\title{
The American College of Rheumatology Preliminary Diagnostic Criteria for Fibromyalgia and Measurement of Symptom Severity
}

\author{
FREDERICK WOLFE, ${ }^{1}$ DANIEL J. CLAUW, ${ }^{2}$ MARY-ANN FITZCHARLES, ${ }^{3}$ DON L. GOLDENBERG, ${ }^{4}$ \\ ROBERT S. KATZ, ${ }^{5}$ PHILIP MEASE, ${ }^{6}$ ANTHONY S. RUSSELL, ${ }^{7}$ I. JON RUSSELL, ${ }^{8}$ JOHN B. WINFIELD, ${ }^{9}$ \\ AND MUHAMMAD B. YUNUS ${ }^{10}$
}

\begin{abstract}
This criteria set has been approved by the American College of Rheumatology (ACR) Board of Directors as Provisional. This signifies that the criteria set has been quantitatively validated using patient data, but it has not undergone validation based on an external data set. All ACR-approved criteria sets are expected to undergo intermittent updates.

As disclosed in the manuscript, these criteria were developed with support from the study sponsor, Lilly Research Laboratories. The study sponsor placed no restrictions, offered no input or guidance on the conduct of the study, did not participate in the design of the study, see the results of the study, or review the manuscript or submitted abstracts prior to the submission of the paper. The recipient of the grant was Arthritis Research Center Foundation, Inc. The authors received no compensation. The ACR found the criteria to be methodologically rigorous and clinically meaningful.

ACR is an independent professional, medical and scientific society which does not guarantee, warrant or endorse any commercial product or service. The ACR received no compensation for its approval of these criteria.
\end{abstract}

Objective. To develop simple, practical criteria for clinical diagnosis of fibromyalgia that are suitable for use in primary and specialty care and that do not require a tender point examination, and to provide a severity scale for characteristic fibromyalgia symptoms.

Methods. We performed a multicenter study of 829 previously diagnosed fibromyalgia patients and controls using physician physical and interview examinations, including a widespread pain index (WPI), a measure of the number of painful body regions. Random forest and recursive partitioning analyses were used to guide the development of a case definition of fibromyalgia, to develop criteria, and to construct a symptom severity (SS) scale.

Results. Approximately $25 \%$ of fibromyalgia patients did not satisfy the American College of Rheumatology (ACR) 1990 classification criteria at the time of the study. The most important diagnostic variables were WPI and categorical scales for cognitive symptoms, unrefreshed sleep, fatigue, and number of somatic symptoms. The categorical scales were summed to create an SS scale. We combined the SS scale and the WPI to recommend a new case definition of fibromyalgia: (WPI $\geq 7$ AND SS $\geq 5$ ) OR (WPI 3-6 AND SS $\geq 9$ ).

Conclusion. This simple clinical case definition of fibromyalgia correctly classifies $88.1 \%$ of cases classified by the ACR classification criteria, and does not require a physical or tender point examination. The SS scale enables assessment of fibromyalgia symptom severity in persons with current or previous fibromyalgia, and in those to whom the criteria have not been applied. It will be especially useful in the longitudinal evaluation of patients with marked symptom variability.

\section{INTRODUCTION}

The introduction of the American College of Rheumatology (ACR) fibromyalgia classification criteria 20 years ago began an era of increased recognition of the syndrome (1). The criteria required tenderness on pressure (tender points) in at least 11 of 18 specified sites and the presence

Supported by Lilly Research Laboratories.

${ }^{1}$ Frederick Wolfe, MD: National Data Bank for Rheumatic Diseases and University of Kansas School of Medicine, Wichita; ${ }^{2}$ Daniel J. Clauw, MD: University of Michigan Medical School, Ann Arbor; ${ }^{3}$ Mary-Ann Fitzcharles, MB, ChB: Montreal General Hospital and McGill University, Montreal, Quebec, Canada; ${ }^{4}$ Don L. Goldenberg, MD: NewtonWellesley Hospital, Tufts University School of Medicine, of widespread pain for diagnosis. Widespread pain was defined as axial pain, left- and right-sided pain, and upper and lower segment pain.

Over time, a series of objections to the ACR classification criteria developed, some practical and some philosophi-

Boston, Massachusetts; ${ }^{5}$ Robert S. Katz, MD: Rush University Medical Center, Chicago, Illinois; ${ }^{6}$ Philip Mease, MD: Seattle Rheumatology Associates and Swedish Medical Center, Seattle, Washington; ${ }^{7}$ Anthony S. Russell, MD: University of Alberta, Edmonton, Alberta, Canada; ${ }^{8}$ I. Jon Russell, MD, PhD: University of Texas Health Sciences Center, San Antonio; ${ }^{9} \mathrm{John}$ B. Winfield, MD: University of North Carolina, Chapel Hill; ${ }^{10}$ Muhammad B. Yunus, MD: The University of Illinois College of Medicine, Peoria. 
cal. First, it became increasingly clear that the tender point count was rarely performed in primary care where most fibromyalgia diagnoses occurred, and when performed, was performed incorrectly (2). Many physicians did not know how to examine for tender points and some simply refused to do so (3). Consequently, fibromyalgia diagnosis in practice has often been a symptom-based diagnosis.

Second, the importance of symptoms that had not been considered by the ACR Multicenter Criteria Committee became increasingly known and appreciated as key fibromyalgia features: for example, fatigue, cognitive symptoms, and the extent of somatic symptoms (4-7). In addition, a number of fibromyalgia experts believed that tender points obscured important considerations and erroneously linked the disorder to peripheral muscle abnormality (8). Finally, some physicians considered that fibromyalgia was a spectrum disorder and was not well served by dichotomous criteria (9).

There was still another important problem with fibromyalgia diagnosis. Patients who improved or whose symptoms and tender points decreased could fail to satisfy the ACR 1990 classification definition. It was not clear how to categorize or assess these patients. In addition, the ACR classification criteria set such a high bar for diagnosis that there was little variation in symptoms among fibromyalgia patients. These two considerations suggested the need for a broad-based severity scale that could differentiate among patients according to the level of fibromyalgia symptoms.

With all of these considerations in mind, we conducted a multicenter study of patients with a diagnosis of fibromyalgia and a control group of rheumatic disease patients with noninflammatory disorders to address the issues of fibromyalgia diagnosis and symptom severity. The objectives of this study were: 1) to identify non-tender point diagnostic criteria for fibromyalgia; these criteria are not

Dr. Clauw has received consultant fees, speaking fees, and/or honoraria (less than $\$ \mathbf{1 0 , 0 0 0}$ each) from Pfizer, Lilly, Forest, Cypress Biosciences, Pierre Fabre, UCB, and AstraZeneca. Dr. Fitzcharles has received consultant fees, speaking fees, and/or honoraria (less than $\$ \mathbf{1 0 , 0 0 0}$ each) from Pfizer, Lilly, and Boehringer Ingelheim, and has given expert testimony for various medicolegal and law firms and received a fee. Dr. Goldenberg has received consultant fees, speaking fees, and/or honoraria (less than $\$ 10,000$ each) from Forest, Pfizer, and Lilly. Dr. Anthony S. Russell has received consultant fees (less than $\$ 10,000$ each) from Amgen/Wyeth, Schering-Plough, UCB, and Abbott Canada, and speaking fees (less than $\mathbf{\$ 1 0 , 0 0 0}$ each) from Roche and BMS. Dr. I. Jon Russell has received consultant fees, speaking fees, and/or honoraria (less than $\$ \mathbf{\$ 0 , 0 0 0}$ each) from Johnson \& Johnson and UCB, and (more than $\$ 10,000$ each) from Pfizer, Lilly, and Jazz. Dr. Winfield has received consultant fees, speaking fees, and/or honoraria (more than $\$ 10,000$ each) from Lilly, Pfizer, Forest, and Jazz, and has given expert testimony for medicolegal and various law firms and received a fee. Dr. Yunus has received speaking fees (more than $\$ 10,000)$ from Forest Laboratories.

Address correspondence to Frederick Wolfe, MD, National Data Bank for Rheumatic Diseases, 1035 North Emporia, Suite 288, Wichita, KS 67214. E-mail: fwolfe@ arthritis-research.org.

Submitted for publication August 24, 2009; accepted in revised form February 10, 2010. meant to replace the ACR classification criteria, but to represent an alternative method of diagnosis; 2) to integrate severity scale-based symptoms in these new clinical criteria, built on the characteristic features of fibromyalgia; these criteria should be suitable for use in primary care and helpful in following patients longitudinally; and 3) to develop a fibromyalgia symptom severity (SS) scale.

To accomplish these objectives, we employed a 2-stage design. In the first stage, we collected an extensive set of patient and physician variables from 514 patients and controls, including an index of pain extent (widespread pain index [WPI]) and characteristic fibromyalgia symptoms. From the resultant data, models were developed for the surrogate classification criteria, diagnostic criteria, and a severity scale. In the second stage, 315 additional patients and controls were assessed by physicians with a reduced set of variables in a physician questionnaire format that could be reduced to a single page suitable for primary care use. The purpose of the phase 2 study was to see if a shortened, practical physician questionnaire would work as well in categorizing fibromyalgia as the longer, more detailed phase 1 assessments. Thus, we primarily report phase 1 data except for comparisons of classification rates and severity variables. Survey criteria, based on patient self-report, will be the subject of a separate report.

\section{SUBJECTS AND METHODS}

Study subjects and physicians. We recruited study physicians by selecting randomly from a list of 113 rheumatologists who were members of the ACR and who indicated an interest in participating in the study after an e-mail solicitation. We also included 5 physicians with known fibromyalgia expertise selected from the authors (FW, DJC, MAF, DLG, ASK, PM, ASR, IJR, JBW).

Participating physicians had to be certain that they would see 10 fibromyalgia patients and 10 noninflammatory controls within a 4-month period. They had to be experienced with fibromyalgia patients and the fibromyalgia tender point examination. All of the physicians completed a short instructional questionnaire on the Internet and satisfactorily completed a brief examination on study requirements and methods. We required that the physician, not an assistant, complete physician assessment forms, and that patient forms could only be completed by the patient.

We required the fibromyalgia study patients to have a previous diagnosis of fibromyalgia. They were enrolled as they appeared in the clinic for usual care (not by being recalled) and without consideration of current diagnosis, severity, or other characteristics. Fibromyalgia subjects must have been diagnosed with fibromyalgia by the same examining rheumatologist prior to the date of study assessment. Patients diagnosed with fibromyalgia could have been diagnosed on clinical grounds or by the ACR classification criteria (10). It was not a requirement of diagnosis to have satisfied the ACR classification criteria. Of the 30 physicians contributing valid patients to phase 1 of the study, 6 used only clinical diagnosis, 9 used only the ACR classification criteria diagnosis, and 15 diagnosed some 
patients using clinical methods and some patients using the ACR methods. Among the expert physicians, 4 used clinical diagnosis, 4 used the ACR classification criteria diagnosis, and 2 used both methods.

Control subjects were patients with noninflammatory painful disorders such as degenerative neck and back pain syndromes or regional disorders, osteoarthritis, tendonitis, or similar disorders who had not been diagnosed previously as having fibromyalgia and who were of the same sex and were no more than 10 years younger or 10 years older than the fibromyalgia case. As with the fibromyalgia patients, the control subjects must have had a prior control diagnosis. Patients with any inflammatory rheumatic disorder (e.g., rheumatoid arthritis), active cancer, fractures, defined neuropathic causes of pain, or other nonrheumatic causes for pain were excluded from the study.

In phase 1, we enrolled 610 patients from 32 referring physicians between December 2, 2008, and April 30, 2009. We excluded 96 patients (15.7\%) for one or more protocol violations. Two physicians enrolled fewer than 10 patients, 1 and 5 patients each, and their patients were excluded because of insufficient site enrollments. We also excluded subjects for invalid control diagnoses $(n=12)$ and for failure to match fibromyalgia patients with controls of the same sex within 10 years of age by sex $(n=96)$ by the time of study closure. After exclusions, there were 514 subjects (mean 17 and median 20 per center). From the group of physician participants there were 10 "experts," defined as having published on fibromyalgia in the medical literature.

Study variables: phase 1 . We instructed study sites to have patients complete their forms before seeing the physician. Physicians were instructed not to look at the patient forms. Physicians' staff was asked to check each patient's form for completeness and request completion of missing items before the patient left the clinic.

Patient variables: phase 1. Patients were asked to indicate in which of 19 body areas they had pain during the last week. These areas were those previously described as part of the Regional Pain Scale (renamed here as the WPI) (11). We also analyzed the WPI as a categorical variable, with categories $0,1,2$, and 3 for values of $0,1-3,4-6$, and $\geq 7$ of the WPI, respectively. The categories were determined by study analyses (see below).

Patients completed 7 categorical scales for symptoms over the past week that were scored as: $0=$ no problem; $1=$ slight or mild problems, generally mild or intermittent; 2 = moderate, considerable problems, often present and/or at a moderate level; and $3=$ severe, pervasive, continuous, life-disturbing problems. Symptoms were assessed using the following words: pain, fatigue, trouble with sleep, trouble with anxiety or depression, problems awaking unrefreshed, and overall severity of your arthritis or fibromyalgia problem. In addition, patients completed 4 visual analog scales that were scored as $0-10$. The scale questions and anchors were 1) severity of pain over the last week, with anchors from no pain to severe pain; 2) how much of a problem has fatigue or tiredness been for you over the past week?, with anchors from fatigue is no prob- lem to fatigue is a major problem; 3) how much of a problem has sleep (i.e., resting at night) been for you in the past week?, with anchors from sleep is no problem to sleep is a major problem; and 4) how much of a problem has waking up unrefreshed been for you in the last week?, with anchors from waking up unrefreshed is no problem to waking up unrefreshed is a major problem. Patients also completed the Health Assessment Questionnaire II functional disability scale (12). Patients indicated the number of medications they used in the last month to help control pain, and reported the extent of morning stiffness.

We also asked patients to indicate which of the following symptoms they experienced in the last 3 months: blurred vision or problems focusing; dry eyes; ringing in ears; hearing difficulties; mouth sores; dry mouth; loss of or change in taste; headache; dizziness; fever; chest pain; shortness of breath; wheezing (asthma); loss of appetite; nausea; heartburn; indigestion or belching; pain or discomfort in the upper abdomen (stomach); liver problems; pain or cramps in the lower abdomen (colon); diarrhea (frequent, explosive watery bowel movements, severe); constipation; black or tarry stools (not from iron); vomiting; joint pain; joint swelling; low back pain; muscle pain; neck pain; weakness of muscles; tiredness (fatigue); depression; insomnia; nervousness (anxiety); seizures or convulsions; trouble thinking or remembering; easy bruising; hives or welts; itching; rash; loss of hair; red, white, and blue skin color changes in fingers on exposure to cold or with emotional upset; sun sensitivity (unusual skin reaction, not sunburn); yellow skin or eyes (jaundice); fluid-filled blisters; numbness/tingling/burning; swelling of the hands, legs, feet, or ankles (not due to arthritis); irritable bowel syndrome; faintness; frequent urination; painful urination; pain, fullness, or discomfort in the bladder region; sensitivity to bright lights, loud noises, or odors; fatigue severe enough to limit daily activity; tender lymph nodes; or frequent sore throats. We summed the positive replies to create a $0-56$ count of somatic symptoms scale.

Physician variables: phase 1. Physicians were asked not to look at the forms completed by the patient. Physicians indicated their certainty of the prior diagnosis on a $0-10$ scale ( $0=$ very uncertain, $10=$ very certain). They performed the ACR tender point count (0-18) (1) and completed the same painful body region scale as their patients did. They completed categorical scales for pain, fatigue, sleep disturbance, cognitive symptoms, waking unrefreshed, and overall (global) severity using the same categorical scoring as the patients did. Physicians also indicated if the patients had the following symptoms: muscle pain, irritable bowel syndrome, fatigue, cognitive problems, muscle weakness, headache, pain/cramps in the abdomen, paresthesias, dizziness, sleep problem, depression, constipation, diarrhea, interstitial cystitis, anxiety, and muscle tenderness.

We then provided the physicians a list of symptoms for reference purposes and asked them to categorize the patients as having few or no somatic symptoms, a moderate number of symptoms, or a great deal of symptoms. The reference list consisted of: muscle pain, irritable bowel 
syndrome, fatigue/tiredness, thinking or remembering problem, muscle weakness, headache, pain/cramps in the abdomen, numbness/tingling, dizziness, insomnia, depression, constipation, pain in the upper abdomen, nausea, nervousness, chest pain, blurred vision, fever, diarrhea, dry mouth, itching, wheezing, Raynaud's phenomenon, hives/welts, ringing in ears, vomiting, heartburn, oral ulcers, loss of/change in taste, seizures, dry eyes, shortness of breath, loss of appetite, rash, sun sensitivity, hearing difficulties, easy bruising, hair loss, frequent urination, painful urination, and bladder spasms.

Based on the study results described below, we created an SS scale by summing the 0-3 scores of somatic symptoms, waking unrefreshed, cognition, and fatigue into a 0-12 scale.

Phase 2 study and variables. The methods and rules for physician and patient recruitment were the same for the second phase, except that the authors with known fibromyalgia expertise were not recruited. In addition, patients did not complete questionnaires. At the time of study closure, 315 valid patients had been enrolled. The phase 2 form was simplified and completed only by the physician. It contained the following items: a categorical WPI and a question about widespread pain. Physicians were provided with a list of the widespread pain regions (scored as $0-3,4-6,7-10$, or $\geq 11$ ), but could not score the individual regions. Physicians performed and recorded a tender point examination; indicated the presence or absence of muscle pain, muscle tenderness, and irritable bowel syndrome; and provided a rating of the extent of somatic symptoms (few or no symptoms, a moderate number of symptoms, or a great deal of symptoms). For reference, a list of 41 symptoms was supplied on the questionnaire. Finally, we included categorical scales for sleep disturbance, unrefreshed sleep, cognitive problems, and fatigue, with scoring as described above.

Statistical methods. On completion, study forms were faxed to the National Data Bank for Rheumatic Diseases, Wichita, Kansa, for processing. They were reviewed for missing data, and if such data were found, the examining physician was contacted immediately for corrections, if possible. Missing patient data and physician data that were not correctable within one week were left as missing. Missing data were rare: in phase $1,81.7 \%$ of patients had no missing data, $12.8 \%$ had 1 missing data point, and $1.6 \%$ had $3-8$ missing data points.

In phase 1 analyses, we considered 3 groups of classifier variables: a short set of variables, an intermediate set, and a complete set. The short set included the WPI and categorical scales for pain, fatigue, sleep disturbance, mood, cognitive problems, somatic symptoms, and unrefreshed sleep. The intermediate set included all of the variables in the short set plus all of the individual somatic symptoms. The complete set included all of the study variables. We anticipated that, because of the need for simplicity, the criteria and severity scales would probably come from the short set. We analyzed the intermediate set under the consideration that the individual symptoms might also be important. The complete set allowed us to compare the 3

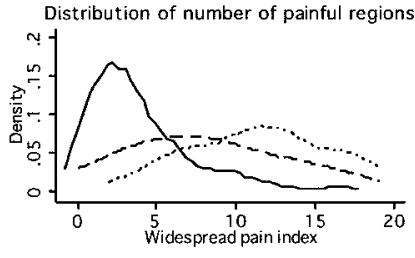

A

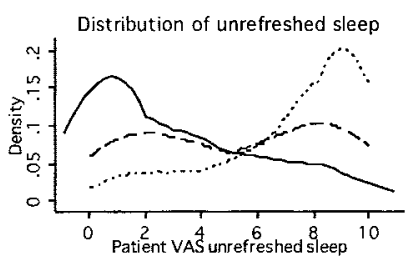

C

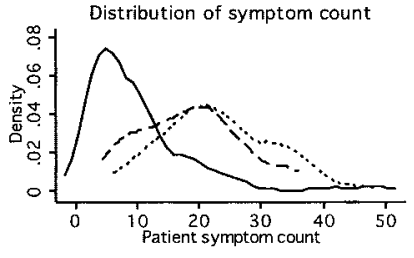

B

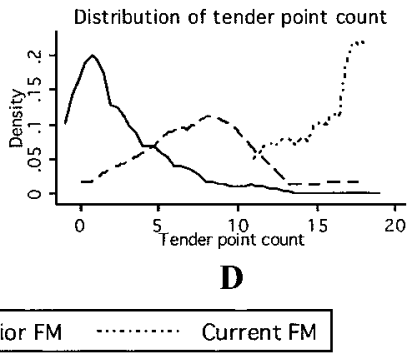

Figure 1. Distribution of key fibromyalgia (FM) variables in controls and patients with current or prior FM (phase 1). VAS = visual analog scale.

sets as to their classifying value and to understand what might be lost in shortening the set of variables.

Comparisons between groups used $t$-tests, regression analyses with dummy variables, and chi-square tests, as indicated. Spearman's coefficients were used for correlation analyses. Data were analyzed using Stata, version 10.1 (StataCorp), and the $\mathrm{R}$ statistical package, version 2.81. Random forest analysis was used to determine variable importance and "out-of-bag" error rates $(10,11)$ using the R statistical package. The out-of-bag error rate is a robust measure of misclassification error. Variable importance is described by the mean decrease in accuracy criterion or the Gini Index, and it represents a ranking of variables in terms of their importance as predictors. The mean decrease in accuracy is thought to be a better measure: “. . . the Gini Index reflects the overall goodness of fit, while the predictive accuracy depends on how well the model actually predicts. The two are related, but they measure different things. Breiman argues that the decrease in predictive accuracy is the more direct, stable and meaningful indicator of variable importance (personal communication)" (13). Classification tree analysis used the rpart recursive partitioning $\mathrm{R}$ analysis programs to determine preliminary cut points for criteria variables (14). Figure 1 describes the distribution of study variables using a probability density function. The reader can think of the probability density function as a "smoothed-out" version of a histogram.

Conflict of interest and ethics. Examining physicians were each compensated $\$ 2,000$ for their work in interviewing, examining, and completing study forms for 10 patients and 10 controls. The study authors received no compensation. The study was approved by the Via Christi Institutional Review Board.

\section{RESULTS}

Demographics. Physicians enrolled 258 valid patients in phase 1 whose clinical diagnosis was fibromyalgia and 
Table 1. Selected clinical characteristics of patients with current or prior fibromyalgia and controls in phase $1 *$

\begin{tabular}{|lccc|}
\hline \multicolumn{1}{c}{ Variable } & $\begin{array}{c}\text { Current } \\
\text { fibromyalgia }\end{array}$ & $\begin{array}{c}\text { Prior } \\
\text { fibromyalgia }\end{array}$ & Controls \\
\hline No. of patients (\%) & $196(38.1)$ & $67(13.0)$ & $251(48.1)$ \\
Widespread pain index (0-19) & $11.4 \pm 4.4$ & $8.2 \pm 5.0$ & $3.8 \pm 3.2$ \\
Physician widespread pain index (0-19) & $11.4 \pm 4.1$ & $7.2 \pm 3.9$ & $3.3 \pm 2.5$ \\
Widespread pain, \% patients & 92.9 & 56.7 & 31.1 \\
Widespread pain, \% physicians & 93.9 & 59.7 & 24.3 \\
Tender point count (0-18) & $15.9 \pm 2.3$ & $7.9 \pm 4.1$ & $2.5 \pm 3.0$ \\
ACR 1990 classification criteria positive, \% patients & 92.9 & 0.0 & 0.0 \\
ACR 1990 classification criteria positive, \% physicians & 93.9 & 0.0 & 0.0 \\
ACR 1990 classification criteria positive, \% patients or & 100.0 & 0.0 & 0.0 \\
physicians & & & 1.5 \\
Physician global severity, categorical (0-3) & 2.1 & 1.8 & 1.4 \\
Patient global severity, categorical (0-3) & 2.4 & $18.2 \pm 8.4$ & $9.7 \pm 8.4$ \\
Patient symptom count (0-48) & $22.9 \pm 8.8$ & $1.9 \pm 0.7$ & $1.2 \pm 0.5$ \\
Physician somatic symptoms (0-3) & $2.3 \pm 0.7$ & $1.0 \pm 0.7$ & $0.7 \pm 0.6$ \\
HAQ-II score (0-3) & $1.3 \pm 0.6$ & $5.2 \pm 3.4$ & $3.1 \pm 3.0$ \\
Patient VAS unrefreshed sleep (0-10) & $7.3 \pm 2.7$ & $4.4 \pm 3.2$ & $3.3 \pm 3.0$ \\
Patient VAS sleep (0-10) & $6.5 \pm 2.8$ & $4.9 \pm 2.7$ & $4.1 \pm 2.8$ \\
Patient VAS pain (0-10) & $6.5 \pm 2.3$ & $5.0 \pm 3.1$ & $3.3 \pm 2.9$ \\
Patient VAS fatigue (0-10) & $7.0 \pm 2.4$ & $6.0 \pm 2.6$ & $3.3 \pm 2.2$ \\
Symptom severity scale (0-12)† & $8.0 \pm 2.6$ & $2.5 \pm 1.4$ & $1.9 \pm 1.9$ \\
No. of pain medications & $3.3 \pm 2.3$ & & \\
\hline * Values are the mean \pm SD unless otherwise indicated. ACR $=$ American College of Rheumatology; HAQ-II = Health \\
Assessment Questionnaire II; VAS = visual analog scale. \\
t Sum of physician somatic symptoms, physician waking unrefreshed, physician cognition, and physician fatigue.
\end{tabular}

256 who were control subjects. Fibromyalgia subjects were slightly older than controls (mean \pm SD age $54.6 \pm 12.9$ versus $52.3 \pm 12.2$ years; $P=0.035$ ), but did not differ by the percentage of males $(8.2 \%$ versus $9.0 \% ; P=0.732)$, percentage of non-Hispanic whites $(86.8 \%$ versus $85.9 \%$; $P=0.770$ ), or education level (mean \pm SD $14.2 \pm 2.1$ versus $14.3 \pm 2.2$ years; $P=0.517$ ).

Diagnosis and diagnostic methods. ACR classification criteria were used in $63.6 \%$ of fibromyalgia diagnoses and clinical diagnosis was used in $36.4 \%$ of fibromyalgia diagnoses. At the time of the study examination, $74.5 \%$ of patients who had been previously diagnosed with fibromyalgia satisfied the ACR classification criteria and $2.0 \%$ of controls satisfied the ACR classification criteria. Based on these data, we categorized patients into 3 groups based on prior diagnosis and ACR classification criteria status: 196 patients (38.1\%) with current fibromyalgia (ACR classification criteria positive, physician fibromyalgia diagnosis positive), 67 patients (13.0\%) with prior fibromyalgia (ACR classification criteria negative, physician fibromyalgia diagnosis positive), and 251 patients (48.1\%) who were neither current nor prior fibromyalgia patients (control subjects) (Table 1). Using a 0-10 physician certainty of prior diagnosis scale, the mean certainties were: fibromyalgia 9.4, prior fibromyalgia 8.7, and control diagnosis 9.1. Patients previously diagnosed by clinical criteria were more likely to be classified as prior fibromyalgia (38.3\%) compared with patients previously diagnosed by the ACR classification criteria $(18.9 \% ; P<0.001)$. The proportion of patients who were controls or had prior or current fibromyalgia did not differ between the group of 10 expert physicians and the 20 clinical rheumatologists $(P=0.640)$.

Characteristics of patients by fibromyalgia status. There was a clear difference in clinical findings and symptom severity among the groups, the current fibromyalgia patients having the greatest symptom severity with prior fibromyalgia generally occupying the severity scale midpoint between current fibromyalgia and controls (Table 1). However, for the count of patient-endorsed somatic symptoms, the physician somatic symptom scale, and the SS scale, prior fibromyalgia patients had scores that were somewhat closer to current fibromyalgia patients than to control subjects. Figure 1 shows differences between groups for key variables. The tender point count (Figure 1D) demonstrates the clearest distinction between groups, followed by unrefreshed sleep (Figure 1C). Prior and current fibromyalgia patients had similar distributions of somatic symptom counts (Figure 1B), while prior fibromyalgia had the WPI shifted somewhat to the left (Figure 1A). Taken as a whole, these data show that approximately $25 \%$ of patients considered to have fibromyalgia by their physicians do not satisfy ACR classification criteria for fibromyalgia, and that they appear to have an intermediate severity position between fibromyalgia patients and control subjects, except for somatic symptoms.

Misclassification rates and fibromyalgia classifiers. To determine variables that best identify fibromyalgia and to examine the predictive power of study variables without the use of tender points, we divided the subjects into ACR 
Table 2. Random forest out-of-bag misclassification rates for physician and patient variable groups in the diagnosis of ACR 1990 classification criteria-positive fibromyalgia (phase 1)*

\begin{tabular}{|c|c|c|c|c|c|}
\hline Variable group & $\begin{array}{c}\text { Error rate } \\
\text { (continuous WPI) }\end{array}$ & $\begin{array}{c}\text { Error rate } \\
\text { (categorical WPI) }\end{array}$ & $\begin{array}{c}\text { Error rate } \\
\text { (no WPI, no } \\
\text { muscle variables) }\end{array}$ & $\begin{array}{c}\text { Error rate } \\
\text { (no WPI, no } \\
\text { muscle tenderness) }\end{array}$ & $\begin{array}{l}\text { Error rate } \\
\text { (no WPI) }\end{array}$ \\
\hline \multicolumn{6}{|c|}{ Physician evaluator, \% } \\
\hline Complete & 6.2 & 6.4 & NA & NA & NA \\
\hline Intermediate & 6.4 & 7.3 & 16.1 & 14.0 & 10.3 \\
\hline Short & 8.9 & 10.3 & 18.4 & NA & NA \\
\hline \multicolumn{6}{|l|}{ Patient evaluator, \% } \\
\hline Complete & 12.7 & NA & & & \\
\hline Intermediate & 14.1 & NA & & & \\
\hline Short & 13.9 & NA & & & \\
\hline
\end{tabular}

classification criteria-positive (all patients satisfying the ACR classification criteria) and ACR classification criterianegative cases (all controls satisfying the ACR criteria), after excluding patients who physicians designated as having fibromyalgia but who did not satisfy the ACR classification criteria. We then used random forest analyses to rank physician predictors of fibromyalgia by their importance and to obtain misclassification rates. We examined the WPI as a continuous variable $(0-19)$ and also after splitting it into categories at $0,1-3,4-6$, and $\geq 7$. In these analyses, we examined the complete set of variables, an intermediate set, and a short set (Table 2).

Depending on the number of variables in the model (variable group) and whether the WPI was used as a continuous or a categorical variable, physician study variables misclassified fibromyalgia cases and controls at rates of $6.2-10.3 \%$ (Table 2). Figure 2A shows that the WPI and muscle tenderness were the most important variables in the classification of cases and noncases. These data provide an estimate of the lowest misclassification rates of surrogate criteria obtainable under optimum data mining conditions.

We next performed analyses to determine misclassification rates when muscle variables (muscle pain, muscle tenderness) and the WPI were removed from the models. Removing all of the muscle variables and the WPI resulted in a misclassification rate of $16.1 \%$ (Table 2). Using the same intermediate model but keeping muscle pain reduced the error rate to $14.0 \%$, whereas removing the WPI but leaving all muscle pain and muscle tenderness variables resulted in a misclassification rate of $10.3 \%$. Using the short variable list (that never contains muscle pain or muscle weakness), the misclassification rate was $18.4 \%$. These data show that misclassification rates obtainable under optimum data mining conditions that do not contain ACR classification criteria-related variables are between $16.1 \%$ and $18.4 \%(14.0 \%$ if muscle pain is allowed).

Based on the information in Table 2, we selected the variables contained in the intermediate WPI categorical model $(7.3 \%$ error rate) for further study because that model represented the best practical model that contained the WPI and muscle data (Table 2) and had the lowest misclassification rate. Random forest analysis provides information on variable importance and misclassification rates, but does not provide information about the optimum cut points for study variables. We then applied recursive partitioning to the data and determined that values of the WPI $\geq 7$ best identified fibromyalgia cases and values $\leq 6$ best identified control cases according to their ACR classification criteria status.

In addition, in the comparison of ACR classification criteria-positive versus ACR classification criteria-negative cases (excluding prior fibromyalgia), the correct classification rates for phase 1 ran between $89.0 \%$ and $92.6 \%$, with higher rates being obtained in phase 2 analyses. When all of the patients were considered, WPI $\geq 7$ performed about as well (83.6\%) as physician diagnosis $(84.1 \%)$ in the classification of fibromyalgia in phase 1 , again with improvement in the phase 2 data. There was some gain obtained by including information about muscle pain or muscle tenderness. Overall, these data show that patients who satisfy the ACR classification criteria can be identified with an acceptable rate of error by the use of the WPI alone, and with very slightly better results if muscle tenderness or muscle pain is assessed.

The SS scale and fibromyalgia diagnostic criteria. To build and identify an alternative definition of fibromyalgia and to create an SS scale, we turned back to the intermediate categorical model that excluded the WPI and muscle variables (Table 2 and Figure 2B). From Figure 2B, we identified 6 categorical rating scale variables that had high importance levels (somatic symptoms, waking unrefreshed, cognition, fatigue, sleep problems, and mood). As shown in Table 3, these variables are strongly correlated with the tender point count and the WPI. For comparison, pain intensity, with correlation coefficients of 0.346 and 0.437, respectively, was less strongly correlated. We created an SS scale by summing the $0-3$ scores of somatic symptoms, waking unrefreshed, cognition, and fatigue, into a 0-12 scale.

When applied to study patients, using the ACR classifi- 

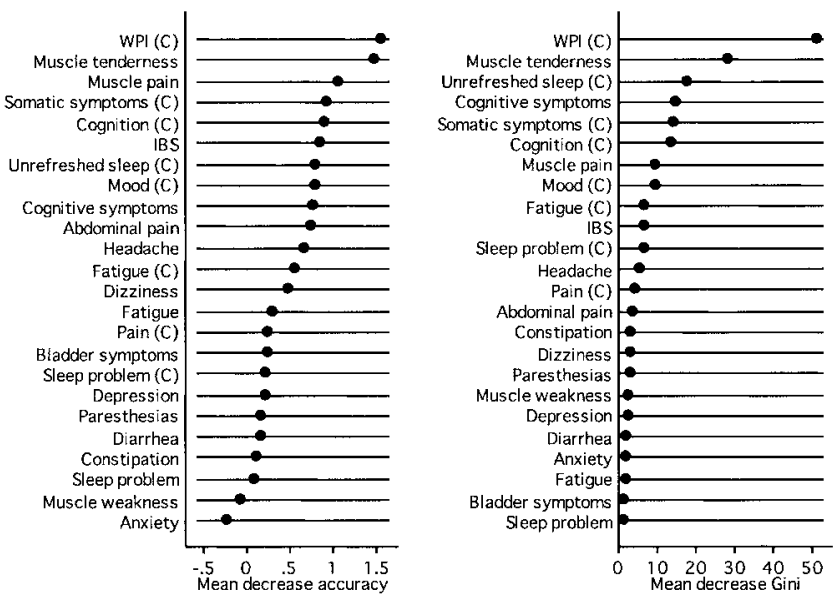

A
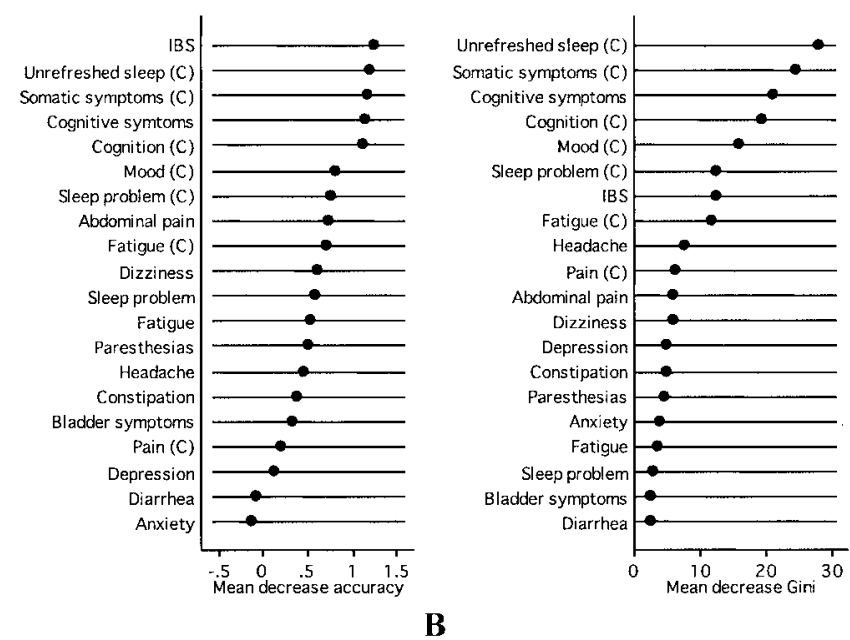

Figure 2. A, Variable importance (physician variables) in distinguishing fibromyalgia from controls in intermediate detail physician evaluation by random forest analysis in phase 1, including the widespread pain index (WPI) and muscle symptoms. B, Variable importance (physician variables) in distinguishing fibromyalgia from controls in intermediate detail physician evaluation in phase 1 by random forest analysis, excluding the Regional Pain Scale (WPI) and muscle symptoms. Other variables are present/ absent conditions. Variable importance is described by the mean decrease in accuracy criterion or the Gini Index, and it represents a ranking of variables in terms of their importance as predictors. The Gini Index reflects the overall goodness of fit, while the predictive accuracy depends on how well the model actually predicts. The two are related, but they measure different things (13). The mean decrease in accuracy is thought to be a better measure. $\mathrm{C}=$ categorical scale; IBS = irritable bowel syndrome.

cation criteria definition of fibromyalgia, the mean \pm SD SS scale score for fibromyalgia was $8.0 \pm 2.6$, for prior fibromyalgia was $6.0 \pm 2.6$, and for controls was $3.3 \pm 2.2$ (Table 1). The SS scale was strongly correlated with the WPI (0.733) and the tender point count (0.680), and was the strongest correlate of WPI after the tender point count, which was correlated with the WPI at 0.773 (Table 3). As shown in Figure 3, the SS scale appropriately follows the definitions of ACR classification criteria-positive fibromyalgia, prior fibromyalgia, and non-fibromyalgia, suggesting that it can categorize fibromyalgia symptom severity.

\begin{tabular}{|c|c|c|}
\hline Variable & $\begin{array}{l}\text { Tender point } \\
\text { count }\end{array}$ & $\begin{array}{c}\text { Widespread } \\
\text { pain index }\end{array}$ \\
\hline Tender point count & 1.000 & 0.773 \\
\hline Widespread pain index & 0.773 & 1.000 \\
\hline Symptom severity scale & 0.680 & 0.733 \\
\hline Symptom severity scale* & 0.672 & 0.713 \\
\hline Somatic symptomst & 0.631 & 0.683 \\
\hline Waking unrefreshedt & 0.622 & 0.656 \\
\hline Cognitiont & 0.575 & 0.592 \\
\hline Fatiguet & 0.548 & 0.604 \\
\hline Sleep problems & 0.524 & 0.546 \\
\hline Mood & 0.504 & 0.534 \\
\hline Pain & 0.346 & 0.437 \\
\hline
\end{tabular}

Fibromyalgia case definition: diagnostic criteria. We used the SS scale and the WPI to recommend a new case definition of fibromyalgia: (WPI $\geq 7$ AND $S S \geq 5$ ) OR (WPI 3-6 AND SS $\geq 9$ ) (Table 4). This definition recognizes that fibromyalgia is more than just a high WPI scale by requiring an SS scale score $\geq 5$, and recognizes that a high level ( $S S \geq 9$ ) of symptoms should be sufficient for diagnosis, provided that there is sufficient body pain. Using this definition, $9.1 \%$ of controls would be diagnosed as having fibromyalgia, $53.1 \%$ of prior fibromyalgia patients would be diagnosed as having fibromyalgia, and $14.1 \%$ of ACR classification criteria-positive cases would not be diagnosed as having fibromyalgia. Overall, the fibromyalgia rate among all of the study subjects would increase from $38.1 \%$ to $45.5 \%$ using the recommended definition. As shown in Table 5, using the ACR classification criteria as the gold standard, the diagnostic criteria diagnose $82.6 \%$ of cases correctly compared with the clinician's correct diagnosis rate of $84.1 \%$.

We also used the SS scale to aid in the diagnosis of

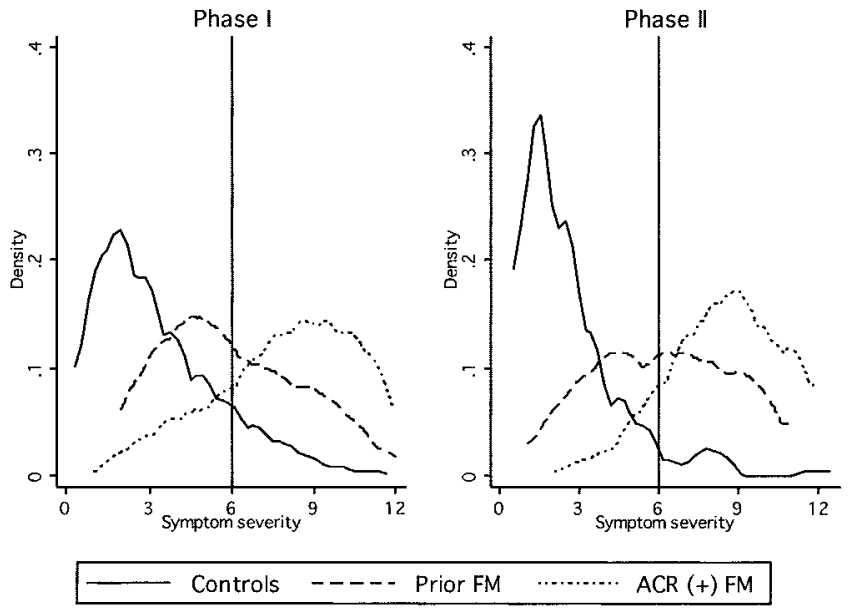

Figure 3. Distribution of severity scores using the American College of Rheumatology (ACR) 1990 classification criteria definition of fibromyalgia (FM) by category of FM diagnosis in phases 1 and 2 . A symptom severity scale score $>6$ identifies patients satisfying the new diagnostic criteria in $92.3 \%$ of cases. 
Table 4. Fibromyalgia diagnostic criteria

Criteria

A patient satisfies diagnostic criteria for fibromyalgia if the following 3 conditions are met:

1) Widespread pain index (WPI) $\geq 7$ and symptom severity (SS) scale score $\geq 5$ or WPI $3-6$ and SS scale score $\geq 9$.

2) Symptoms have been present at a similar level for at least 3 months.

3) The patient does not have a disorder that would otherwise explain the pain.

Ascertainment

1) WPI: note the number areas in which the patient has had pain over the last week. In how many areas has the patient had pain? Score will be between 0 and 19.
Shoulder girdle, left
Shoulder girdle, right
Hip (buttock, trochanter), left
Hip (buttock, trochanter), right
Upper leg, left
Upper leg, right
Jaw, left
Jaw, right
Chest
Upper back
Upper arm, left
Upper arm, right
Lower leg, left
Abdomen
Lower arm, left
Lower arm, right
Lower leg, right
Lower back
Neck
2) SS scale score:
Fatigue
Waking unrefreshed
Cognitive symptoms
For the each of the 3 symptoms above, indicate the level of severity over the past week using the following scale:
$0=$ no problem
$1=$ slight or mild problems, generally mild or intermittent
2 = moderate, considerable problems, often present and/or at a moderate level
3 = severe: pervasive, continuous, life-disturbing problems
Considering somatic symptoms in general, indicate whether the patient has:*

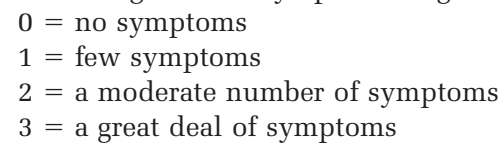

The SS scale score is the sum of the severity of the 3 symptoms (fatigue, waking unrefreshed, cognitive symptoms) plus the extent (severity) of somatic symptoms in general. The final score is between 0 and 12.

* Somatic symptoms that might be considered: muscle pain, irritable bowel syndrome, fatigue/tiredness, thinking or remembering problem, muscle weakness, headache, pain/cramps in the abdomen, numbness/tingling, dizziness, insomnia, depression, constipation, pain in the upper abdomen, nausea, nervousness, chest pain, blurred vision, fever, diarrhea, dry mouth, itching, wheezing, Raynaud's phenomenon, hives/welts, ringing in ears, vomiting, heartburn, oral ulcers, loss of/change in taste, seizures, dry eyes, shortness of breath, loss of appetite, rash, sun sensitivity, hearing difficulties, easy bruising, hair loss, frequent urination, painful urination, and bladder spasms.

clinical fibromyalgia, because Figure 3 suggests that severity scale criteria might be applicable. A severity score $\geq 7$ correctly classifies $92.3 \%$ of cases identified by the new diagnostic criteria definition: (WPI $\geq 7$ AND SS $\geq 5$ ) OR (WPI 3-6 AND SS $\geq 9$ ). When applied to the ACR classification criteria, correct classification falls to $79.2 \%$. This

Table 5. Percentage of patients correctly classified according to ACR 1990 classification criteria status and diagnostic criteria*

\begin{tabular}{|c|c|c|c|c|}
\hline \multirow[b]{2}{*}{ Comparison } & \multicolumn{2}{|c|}{ Phase 1} & \multicolumn{2}{|c|}{ Phase 2} \\
\hline & $\begin{array}{l}\text { Prior FM } \\
\text { excluded }\end{array}$ & $\begin{array}{c}\text { All } \\
\text { patients }\end{array}$ & $\begin{array}{l}\text { Prior FM } \\
\text { excluded }\end{array}$ & $\begin{array}{c}\text { All } \\
\text { patients }\end{array}$ \\
\hline ACR classification criteria positive, FM patients & & 74.0 & & 75.6 \\
\hline \multicolumn{5}{|l|}{$\begin{array}{l}\text { Classifying patients according to ACR } 1990 \\
\text { classification criteria status }\end{array}$} \\
\hline ACR 1990 criteria vs. physician diagnosis & & 84.1 & & \\
\hline ACR 1990 criteria vs. WPI $\geq 7$ & 89.0 & 83.6 & 92.3 & 87.9 \\
\hline ACR 1990 criteria vs. WPI $\geq 7$ plus muscle pain & 89.9 & 84.6 & 95.2 & 90.5 \\
\hline ACR 1990 criteria vs. WPI $\geq 7$ plus muscle tenderness & 92.6 & 87.4 & 95.2 & 90.5 \\
\hline \multicolumn{5}{|l|}{ FM diagnostic criteria } \\
\hline $\begin{array}{l}\text { ACR } 1990 \text { classification criteria vs. diagnostic criteria } \\
\quad((W P I \geq 7 \text { AND } S S \geq 5) \text { OR } S S \geq 9)\end{array}$ & 88.1 & 82.6 & 95.2 & 90.8 \\
\hline $\begin{array}{l}\text { ACR } 1990 \text { classification criteria vs. categorical SS } \\
\text { scale (SS } \geq 7 \text { ) }\end{array}$ & 84.3 & 79.2 & 88.8 & 84.5 \\
\hline $\begin{array}{l}\text { Diagnostic criteria ((WPI } \geq 7 \text { AND } S S \geq 5) \text { OR } S S \geq 9) \\
\text { vs. categorical SS scale }(S S \geq 7)\end{array}$ & & 92.3 & & 89.2 \\
\hline
\end{tabular}


falloff is to be expected, as the definition of fibromyalgia is shifted in the new criteria definition. Using the newly proposed categorical definition for fibromyalgia, the mean \pm SD SS scale score for fibromyalgia was $8.5 \pm 2.0$, and for non-fibromyalgia was $2.9 \pm 1.6$. Using the SS scale score cutoff of $\geq 7$, the mean \pm SD respective scores were $8.6 \pm 1.8$ and $2.8 \pm 1.4$.

Phase 2 validation. The ACR classification criteria were used in $74.6 \%$ of fibromyalgia diagnoses. Among patients diagnosed as having fibromyalgia, 76.0\% met ACR classification criteria compared with $74.5 \%$ in the first phase of the study. We categorized the 315 patients into 3 groups based on prior diagnosis and current ACR classification criteria status: $42.2 \%$ with current fibromyalgia, $13.3 \%$ with prior fibromyalgia, and $44.4 \%$ who were neither current nor prior fibromyalgia patients. The validation sample was slightly different in the 2 phases (Figure 3), and criteria to identify ACR classification criteria cases worked slightly better in phase 2 .

\section{DISCUSSION}

We are aware of the inherent problems in the diagnosis of somatic syndromes that lack objective physical or laboratory features or well-characterized pathologic findings. The utility of such diagnostic criteria is very dependent on the clinical setting. The initial ACR fibromyalgia classification criteria stipulated that chronic widespread pain was present for 3 months and it was understood that no other disease was thought by the examiner to be accounting for the chronic widespread pain. These ACR classification criteria performed well in specialty clinics and were very useful in providing some patient homogeneity for clinical trials. However, they have not been widely embraced in primary care.

The diagnostic criteria suggested here (Table 4) are not meant to replace the ACR classification criteria. Instead, they were designed to address certain realities. First, the clinical diagnosis in primary care does not ordinarily involve a tender point count or an adequately executed tender count when performed. Second, the case definition of fibromyalgia has changed somewhat with increasing recognition of the importance of cognitive problems and somatic symptoms, factors that were not even considered in the 1990 ACR classification criteria.

In developing new diagnostic criteria, we identified 2 variables that best defined fibromyalgia and its symptom spectrum: the WPI and the composite SS scale. The WPI, which strongly correlated with the tender point count and the SS scale, best identified patients diagnosed with the ACR classification criteria. We also selected the SS scale, a composite variable composed of physician-rated cognitive problems, unrefreshed sleep, fatigue, and somatic symptom count to measure fibromyalgia symptom severity. We used the WPI and the SS scale together to define fibromyalgia diagnostic criteria: (WPI $\geq 7$ AND SS $\geq 5$ ) OR (WPI 3-6 AND SS $\geq 9$ ). We also used the SS scale alone to provide a measure of fibromyalgia symptom severity. In many respects, these criteria are similar in concept to those proposed by Yunus et al in 1981 (15). These authors allowed fewer tender points in the presence of many symptoms, and they stressed the importance of symptoms.

One of the important findings of the current study was that approximately $25 \%$ of diagnosed fibromyalgia patients in both phases of the study did not satisfy the ACR classification criteria, although they were considered to have fibromyalgia by their physicians. Neither the ACR classification criteria nor the diagnostic criteria suggested here provide a solution for this classification dilemma. It arises because fibromyalgia diagnosis is based on severity assessments. The loss of a tender point or a painful region for any reason, including improvement, can result in failing to meet classification or diagnostic criteria. Practically, the conundrum involves patients who have fibromyalgia but who do not meet criteria versus patients who do not have fibromyalgia because they do not meet criteria. In this respect, fibromyalgia differs from rheumatoid arthritis or systemic lupus erythematosus, illnesses that do not involve diagnosis based on symptom severity, where patients continue to have their condition even though they may subsequently not meet diagnostic criteria.

We suggest the use of the SS scale to quantify fibromyalgia symptom severity as a workable solution to this problem. As shown in Figure 3, the distribution of SS scale scores defines the 3 diagnostic categories and provides measurement of fibromyalgia symptom severity to patients who do and do not currently satisfy ACR fibromyalgia criteria. Practically, patients who have satisfied the ACR classification or diagnostic criteria at one time can be followed with the symptom scale, thereby linking current status to previous diagnosis.

In creating the SS scale we chose not to use the mood variable, although it had importance in Figures 2A and B. We chose not to include mood because we judged mood difficult to assess and because it might be a resultant feature rather than a primary feature of the illness. Although we omitted mood, it was correlated with the SS scale at 0.725 and with the components of the SS scale as follows: cognition 0.625, somatic symptoms 0.621, waking unrefreshed 0.619, and fatigue 0.612. In addition, as shown in Table 3, a 5-component SS scale that included mood did not perform as well as a 4-component scale. Unrefreshed sleep was a better measure than sleep disturbance and we recommend its use in the SS scale. However, in sensitivity analyses, it provided marginally better differentiating power; we suggest that either scale can be used, although unrefreshed sleep is better. Of the binary variables, irritable bowel syndrome, abdominal pain, and headache had variable importance. However, they added no power to correct classification and we did not include them in the diagnostic criteria.

In addition, in developing criteria for clinical diagnosis we were cognizant of the need to make the diagnostic criteria simple enough and easy enough so that they would actually be used in clinical practice. To that end, we used simple categorical scales for the symptom scale and compressed the WPI to a similar categorical scale. None of this guarantees that the scales will be used appropriately, but it is easy enough to use them appropriately, and the knowl- 
edge that these are key factors will increase recognition of such important symptoms.

In designing this study, we were concerned that a simple questionnaire would lose too much information. Therefore, we used a complex series of questions and evaluation in phase 1. Based on the results in Table 2, which showed little gain from the complete data sets, we collapsed the questionnaire to categorical scales and changed the format to a form that could be used in the clinic. Results from phase 2 in Table 5 suggest that the criteria would work satisfactorily in the final format, which is included in Table 4.

Readers might wonder: why is it so difficult to make new fibromyalgia criteria? The central problem in fibromyalgia criteria is the absence of a gold standard or case definition. The ACR classification criteria, rightly criticized for circularity (16), created a de facto case definition by imposing the 11 tender point rules on top of a crude definition of widespread pain (17). Subsequent studies as well as the data of this study showed the importance of (improved) quantitative measures of body pain (18-22), and of the key variables that comprise the SS scale in characterizing fibromyalgia. The shift in the conceptualization of fibromyalgia that occurred in the clinic and in research studies, however, provided no clear case definition. In the current study, we derived an empirical case definition from the variable importance analyses. The diagnostic criteria and SS scale we have proposed shift the fibromyalgia definition somewhat toward important symptoms: first, the criteria can be satisfied by a high level of symptoms if the WPI score is not high enough; second, fibromyalgia symptoms are accorded appropriate importance by the provision of the SS scale.

The suggested diagnostic criteria create a small discordance between the older ACR classification criteria case definition and the current definition that we have proposed. However, it is clear that the ACR classification criteria are unable to provide a useful classification for the $25 \%$ of fibromyalgia cases we identified as prior fibromyalgia, something that can be somewhat ameliorated through the use of the SS scale. In addition, it should be remembered that physician diagnosis only correctly classified $84.1 \%$ of cases, while the proposed diagnostic criteria, even with shift of definition, identified $82.6 \%$ of patients correctly (Table 5).

We envision the use of the diagnostic criteria in the following ways: following a diagnosis of fibromyalgia by ACR classification or diagnostic criteria, the results of the SS scale can document baseline symptom severity. Should the patient subsequently not satisfy ACR classification or diagnostic criteria, the SS scale can be used to measure current symptom severity status and change in status without the contradiction of having a diagnosis of fibromyalgia but not satisfying fibromyalgia criteria at the same time. Of course, the SS scale can also be used at any time regardless of diagnostic status. The SS scale alone provides some diagnostic information, but does not include the WPI. It provides information as to symptom severity and it allows fibromyalgia to be seen as part of a continuum, as some have suggested. The criteria and severity scale also provide room for those who are uncomfortable with the fibro- myalgia concept, as they can simply report the WPI and the SS scale.

Physicians who are used to the ACR classification criteria may be uncomfortable with the absence of a physical examination criterion in the new diagnostic criteria. However, $98.7 \%$ of the patients in the study satisfying the diagnostic criteria had a least 1 tender point and $96.3 \%$ had 3 or more tender points. Even though the new criteria do not include a physical examination criterion, all of the patients being diagnosed should have a physical examination, which may include examination of tender point sites. We would like to point out that implicit in the 1990 ACR classification criteria was the requirement that clinical examination and clinical judgment had excluded other causes of chronic widespread pain, and such an exclusion is also implicit in the proposed diagnostic criteria. It is important for physicians to perform an appropriate clinical assessment to exclude other diagnoses, and/or to identify potential coexisting rheumatic diseases that may require treatment themselves.

Although not a study requirement for enrollment, all of the patients with fibromyalgia diagnosed by physicians had symptoms for at least 3 months. However, the timeframe for the physician assessment of WPI and the SS scale was 1 week. We used this timeframe to more accurately determine the level of symptoms. The new diagnostic criteria require that patients diagnosed as having fibromyalgia will have had a similar level of symptoms for at least 3 months, in agreement with the ACR classification criteria.

Although we collected data directly from patients as well as data from physicians, patient data did not work as well in correct classification as physician data (Table 2). The best misclassification rate obtainable with patient data was $12.7 \%$ compared with $6.2 \%$ with physician data. This difference is related to the fact that it is the physician and not the patient who makes the diagnosis.

This study has a number of limitations. Although our goal was to develop simple, practical criteria for clinical diagnosis of fibromyalgia that are suitable for use in primary and specialty care, we did not study the performance of these criteria in primary care. We recommend that a followup study in the primary care setting be accomplished. We also did not test the criteria among those with other rheumatic conditions, and we recommend that this be done in the future, too. The patient population should include those with relevant differential diagnoses (i.e., other rheumatic conditions) to determine the rate of misclassification that may occur. If, as we expect, the diagnostic criteria perform well, it seems possible that the ACR classification criteria might be withdrawn.

In summary, we have developed a case definition and diagnostic criteria for fibromyalgia: (WPI $\geq 7$ AND SS $\geq 5$ ) OR (WPI 3-6 AND SS $\geq 9$ ). This simple clinical case definition of fibromyalgia correctly classifies $88.1 \%$ of cases classified by the ACR classification criteria, and does not require a physical or tender point examination. The SS scale enables assessment of fibromyalgia symptom severity in persons with current or previous fibromyalgia, and in those to whom the criteria have not been applied. It will be especially useful in the longitudinal evaluation of patients with marked symptom variability. 


\section{ACKNOWLEDGMENTS}

The authors thank Kimberly Harp of the National Data Bank for Rheumatic Diseases for her superb work with study physicians and their questionnaire submissions that insured study quality. The following physicians participated in the study by enrolling patients and performing evaluations: Micha Abeles, MD, Fu Flora Bai, MD, Jane H. Box, MD, Robert G. Carpenter, MD, Kimberly Carter Cerveny, MD, John J. Condemi, MD, Kathryn H. Dao, MD, Kathleen P. Flint, MD, Neil J. Gonter, MD, Sivalingam Kanagasegar, MD, Wendy W. Lee, MD, Robert W. Levin, MD, Michael W. Lindamood, MD, MPH, Ndudi Oparaeche, MD, Elizabeth Reinitz, MD, Russell R. Rothenberg, MD, Amjad Roumany, MD, Stuart L. Silverman, MD, FACP, FACR, Ekaterina V. Soforo, MD, Terence W. Starz, MD, Roland Staud, MD, Nicholas R. Straniero, Jr., MD, David H. Trock, MD, Sudhakar Tumuluri, MD, Peter D. Utsinger, MD, Corey W. Walker, MD, John B. Winfield, MD, Ibrahim S. Alghafeer, MD, Jeff A. Alloway, MD, Philip A. Baer, MD, FRCPC, Lan X. Chen, MD, PhD, Ara H. Dikranian, MD, Steven J. Golombek, MD, Andrew M. Jasek, MD, Alan J. Kivitz, MD, CPI, Sri L. Koneru, MD, Theresa A. Lawrence-Ford, MD, Frances Y. Leung, MD, FRCPC, Robert J. Meador, Jr., MD, Sherif A. Nasef, MD, Rakesh C. Patel, DO, Lynne S. Peterson, MD, Karina D. Torralba, MD, Anthony M. Turkiewicz, MD, Brian T. Walitt, MD, MPH, William S. Wilke, MD, and Karen E. Zagar, MD.

\section{AUTHOR CONTRIBUTIONS}

All authors were involved in drafting the article or revising it critically for important intellectual content, and all authors approved the final version to be published. Dr. Wolfe had full access to all of the data in the study and takes responsibility for the integrity of the data and the accuracy of the data analysis.

Study conception and design. Wolfe, Clauw, Fitzcharles, Goldenberg, Katz, Anthony S. Russell, I. Jon Russell, Winfield, Yunus. Acquisition of data. Wolfe, Clauw, Fitzcharles, Goldenberg, Katz, Mease, Anthony S. Russell.

Analysis and interpretation of data. Wolfe, Clauw, Goldenberg, Mease, Anthony S. Russell, I. Jon Russell, Winfield, Yunus.

\section{ROLE OF THE STUDY SPONSOR}

Lilly Research Laboratories did not participate in the design of the study, see the results of the study, or review the manuscript or submitted abstracts.

\section{REFERENCES}

1. Wolfe F, Smythe HA, Yunus MB, Bennett RM, Bombardier C, Goldenberg DL, et al. The American College of Rheumatology 1990 criteria for the classification of fibromyalgia: report of the Multicenter Criteria Committee. Arthritis Rheum 1990;33: $160-72$.

2. Fitzcharles MA, Boulos P. Inaccuracy in the diagnosis of fibromyalgia syndrome: analysis of referrals. Rheumatology (Oxford) 2003;42:263-7.

3. Buskila D, Neumann L, Sibirski D, Shvartzman P. Awareness of diagnostic and clinical features of fibromyalgia among family physicians. Fam Pract 1997;14:238-41.

4. Mease P, Arnold LM, Choy EH, Clauw DJ, Crofford L, Glass $\mathrm{JM}$, et al, for the OMERACT Fibromyalgia Working Group. Fibromyalgia syndrome module at OMERACT 9: domain construct. J Rheumatol 2009;36:2318-29.

5. Choy EH, Arnold LM, Clauw DJ, Crofford LJ, Simon LS, Martin SA, et al. Content and criterion validity of the preliminary core dataset for clinical trials in fibromyalgia syndrome. J Rheumatol 2009;36:2330-4.

6. Mease P. Fibromyalgia syndrome: review of clinical presentation, pathogenesis, outcome measures, and treatment. J Rheumatol Suppl 2005;75:6-21.

7. Mease P, Arnold LM, Bennett R, Boonen A, Buskila D, Carville S, et al. Fibromyalgia syndrome. J Rheumatol 2007;34: 1415-25.

8. Crofford LJ, Clauw DJ. Fibromyalgia: where are we a decade after the American College of Rheumatology classification criteria were developed? [editorial]. Arthritis Rheum 2002;46: 1136-8.

9. Wolfe F, Michaud K. Outcome and predictor relationships in fibromyalgia and rheumatoid arthritis: evidence concerning the continuum versus discrete disorder hypothesis. J Rheumatol 2009;36:831-6.

10. Wolfe F. When to diagnose fibromyalgia. Rheum Dis Clin North Am 1994;20:485-501.

11. Wolfe F. Pain extent and diagnosis: development and validation of the regional pain scale in 12,799 patients with rheumatic disease. J Rheumatol 2003;30:369-78.

12. Wolfe F, Michaud K, Pincus T. Development and validation of the Health Assessment Questionnaire II: a revised version of the Health Assessment Questionnaire. Arthritis Rheum 2004;50:3296-305.

13. Berk RA. Data mining within a regression framework. In: Maimon O, Rokach L, editors. Data mining and knowledge discovery handbook. New York: Springer; 2005. p. 231-55.

14. Atkinson EJ, Therneau TM. An introduction to recursive partitioning using the RPART routines. Rochester: Mayo Foundation; 2000.

15. Yunus M, Masi AT, Calabro JJ, Miller KA, Feigenbaum SL. Primary fibromyalgia (fibrositis): clinical study of 50 patients with matched normal controls. Semin Arthritis Rheum 1981; 11:151-71.

16. Cohen ML, Quintner JL. Fibromyalgia syndrome, a problem of tautology. Lancet 1993;342:906-9.

17. Macfarlane GJ, Croft PR, Schollum J, Silman AJ. Widespread pain: is an improved classification possible? J Rheumatol 1996;23:1628-32.

18. Macfarlane GJ. Chronic widespread pain and fibromyalgia: should reports of increased mortality influence management? Curr Rheumatol Rep 2005;7:339-41.

19. Moldofsky $\mathrm{H}$. The significance of the sleeping-waking brain for the understanding of widespread musculoskeletal pain and fatigue in fibromyalgia syndrome and allied syndromes. Joint Bone Spine 2008;75:397-402.

20. Hieblinger R, Coenen M, Stucki G, Winkelmann A, Cieza A. Validation of the International Classification of Functioning, Disability and Health core set for chronic widespread pain from the perspective of fibromyalgia patients. Arthritis Res Ther 2009;11:R67.

21. Macfarlane GJ, Jones GT, Knekt P, Aromaa A, McBeth J, Mikkelsson $\mathrm{M}$, et al. Is the report of widespread body pain associated with long-term increased mortality? Data from the Mini-Finland Health Survey. Rheumatology (Oxford) 2007; 46:805-7.

22. Macfarlane GJ, Pye SR, Finn JD, Wu FC, Silman AJ, Bartfai G, et al. Investigating the determinants of international differences in the prevalence of chronic widespread pain: evidence from the European Male Ageing Study. Ann Rheum Dis 2009; 68:690-5. 